\title{
Episodic affordances contribute to language comprehension
}

ARTHUR M. GLENBERG, RAYMOND BECKER, SUSANN KLÖTZER, LIDIA KOLANKO, SILVANA MÜLLER, AND MIKE RINCK*

Arizona State University

Wilfrid Laurier University

Dresden University of Technology

Radboud University Nijmegen

\section{Abstract}

We demonstrate how a particular type of knowledge about objects, their spatial locations and thus how to direct actions toward them, contributes to the comprehension of language about those objects. In four experiments, participants judged if sentences were about normal objects (e.g., "The apple has a stem") or odd objects (e.g., "The apple has an antenna"). The Normal response key was either on the left of a response box or on the right. The named objects were themselves either on the left or the right of the response box. We demonstrate a compatibility effect in which responding Normal to the side where the object was located was faster than responding Normal to the opposite side. Furthermore, this effect was equally strong for sentences describing states of the objects (as above) and sentences describing actions (e.g., "Touch the apple at the stem"); the compatibility effect was found when the objects were removed; the effect required compatibility between actions, not just spatial locations; and the effect was found in both English and German. The results are discussed in relation to how action systems are used in language comprehension.

Keywords

language comprehension, reading, affordances, embodiment, ACE

* Correspondence address: Arthur Glenberg, Department of Psychology, Arizona State University, 950 S. McAllister, Tempe, AZ 85287, USA. E-mail: glenberg@asu.edu. This research was supported by NSF grants BCS-0315434 and INT-0233175, and Department of Education grant R305H030266 to Arthur Glenberg, and a grant from the German Academic Exchange Service (DAAD) to Mike Rinck. The opinions expressed in this paper are those of the authors and do not necessarily reflect those of the funding agencies. 


\section{A. M. Glenberg et al.}

\section{Introduction}

What sort of object knowledge contributes to the comprehension of language about those objects? Here we investigate one type of knowledge that we characterize as episodic affordances: temporary characteristics of objects, such as spatial location, that determine how to interact with those objects. For example, where an object is located helps to determine how to look at it, how to grasp it, and how to move toward or away from it. We call these sorts of affordances "episodic" because they can change from moment to moment (as the location of the object or the actor changes), as opposed to more permanent affordances such as the fact that a cup affords drinking. We begin by reviewing the notion of affordances in language comprehension. Next, we present four experiments that demonstrate the use of episodic affordances during language comprehension. These experiments reveal that episodic affordances play a role in language comprehension a) when sentences describe actions and when they describe states, irrespective of the specific language used, b) when the objects are visible in the environment and when they are not, c) when real actions correspond closely to described actions, and d) that the effect is more strongly associated with information about action than information about spatial location.

\subsection{Affordances in language comprehension}

The notion of affordances was developed by Gibson (e.g., 1979) to characterize the interaction between types of bodies and types of objects. That is, an object such as a chair affords some interactions (e.g., sitting, but not swimming) for some types of bodies (e.g., humans, but not dolphins). Particulars of the body may be important, in that, for example, a chair may afford hiding for a child but not an adult. There are four sources of support for the claim that affordances play a role in language comprehension: logical and theoretical, data from neuroscientific investigations of language, studies of eye movements, and other behavioral data.

Consider the logical and theoretical grounds first. Searle (1980) developed the case that the abstract, amodal, and arbitrary symbols of language (i.e., words) can only be meaningful when those symbols are grounded in something outside of the symbol system. Without that grounding, words, like mathematical symbols (e.g., $\mathrm{X}=2 \mathrm{Y}$ ), can equally well refer to an infinity of objects and relations with no way to constrain them to the particular situation a speaker or writer wishes to communicate about (see also Putnam, as cited in Lakoff 1987).

Glenberg and Robertson (1999, 2000) demonstrated the necessity of grounding in language comprehension tasks and developed the Indexical 
Hypothesis $(\mathrm{IH})$ as a means of describing how the symbols of language become grounded. According to the $\mathrm{IH}$, words and phrases are indexed, or mapped, onto objects in the environment or perceptual symbols (Barsalou 1999), which are analog representations extracted from perceptual experience. Next, affordances are derived from the objects or perceptual symbols (Borghi et al. 2004; Glenberg and Robertson 2000; Kaschak and Glenberg 2000). Finally, the affordances are combined or meshed (integrated in a manner that respects bodily and physical constraints on action) as directed by syntactic constructions (Coventry and Garrod 2003; De Vega et al. 2004; Kaschak and Glenberg 2000). On this account, language understanding requires the integration of affordances, not simply the alignment of words. The product of the mesh process is grounded in perception and action, and it has the further benefit of directly guiding action in the world.

The proposition that affordances are considered during language comprehension is consistent with several lines of evidence from neuroscience. For example, Hauk and colleagues (2004) used fMRI to examine differential activation of brain regions while people listened to verbs such as "lick," "pick," and "kick." The verbs selectively activated areas of the motor cortex corresponding to the actions the verbs described. Buccino and colleagues (2005) measured electrical activity in hand and leg muscles as people listened to sentences describing activities normally utilizing hands or legs. When transcranial magnetic stimulation of corresponding areas of the motor cortex was used, the measured electrical activity (in the effector muscles) was sensitive to the sentence content (see also, Glenberg et al. 2008). Finally, Rizzolatti and Arbib (1998) have speculated about the evolutionary development of language from the mirror neuron system (which includes an area of monkey brain, F5, homologous to Broca's area in humans). Mirror neurons appear to be used in recognizing the actions of conspecifics as the same as actions undertaken by the self (Buccino et al. 2004). Thus, the mirror neuron system has been linked to language, gesture, and actions, providing at least one source for a link between language and affordances.

Work examining eye movements during language comprehension is also consistent with a link between language comprehension and affordances. Chambers and colleagues (2002) recorded eye movements while people listened to (and then acted on) commands to move various objects displayed in front of the listener, equivalent to: Put the $\mathrm{X}$ into the $\mathrm{Y}$. Upon hearing "into", the eyes would preferentially move to a container that afforded the action even before the container was mentioned. Kamide and colleagues (2003) recorded eye movements to objects in a picture while people listened to sentences such as "The woman will slide the butter to 
the man" or "The woman will spread the butter on the bread." For the "spread" sentence, there were more anticipatory eye movements to a picture of bread (an object that easily affords spreading) than for the "slide" sentence.

A particularly compelling demonstration of the embodiment of language comprehension was reported by Chambers and colleagues (2004). Participants listened to sentences describing an action, and then they took the relevant action. While listening and acting, the participant's eye movements were monitored. Consider the following situation. The participant was facing four items, a whistle on a plate, an empty box, an empty folder, and a whistle (with a lanyard) on a folder. The participant listened and acted on two types of sentences. (1) "Put the whistle that's on the folder in the box," and (2) "Put the whistle on the folder in the box". Sentence (2) uses a reduced relative clause (the "that's on" is eliminated). Sentence (2) is perfectly acceptable in some situations, namely when there are two whistles in the environment that need to be differentiated. In this case, a relative clause ("that's on the folder" or the reduced "on the folder") is expected because it differentiates between the two whistles. If there had been only one whistle, however, then Sentence (2) is temporarily ambiguous. That is, while listening to the beginning of the sentence, "Put the whistle on the folder..." one could reasonably interpret "on the folder" as the place to put the one whistle. In fact, when there were two whistles, participants spent little time looking at the empty folder, presumably because the relative clause (full or reduced) was expected.

In another condition of the experiment, the participants were given a hook to hold, and they were required to use the hook to carry out the instructions. In this condition, there was functionally only one whistle because only the whistle with the lanyard affords picking up and moving with the hook. In this condition when people heard Sentence (2), they spent substantially more time looking at the empty folder than when they heard Sentence (1). The interpretation of this effect is quite striking: Holding the hook changes the affordances of the situation so that functionally there is only one whistle. This change in affordances changes the preferred parsing of the reduced relative clause so that "... on the folder" is interpreted as a goal location (hence the time spent looking at the empty folder) rather than a relative clause differentiating the two whistles. Furthermore, Chambers and colleagues (2004) demonstrated that the effects of changing the affordances were observable as soon as $150 \mathrm{msec}$. after the word "whistle" was uttered. In other words, the affordances affected the immediate, on-line parsing of the sentence.

A many behavioral studies are consistent with the hypothesis that language comprehension taps information about affordances. Borghi and 
colleagues (2004) had participants verify that an object named in a sentence (e.g., "There is a car in front of you") has various parts (e.g., roof or wheel). Half of the parts were near the top of the object (e.g., roof) and would require movements in an upward direction to interact with those parts, whereas the other half of the parts were located near the bottom of the object (e.g., wheel). In one condition, to respond "Yes, the object has the part" the participant was required to move to a response button that required an upward movement, and in another condition the Yes response required a downward movement. People were faster to respond Yes when the direction of movement was consistent with the direction needed to interact with the named part of the object. Furthermore, this Action-sentence Compatibility Effect (ACE) was greater when movement to the button was required rather than simply pressing buttons in a compatible spatial location, thus implicating action-based information and not simply a spatial code. Apparently, simply considering an object, even without any intent to act, leads to the derivation of affordances (see also, Tucker and Ellis 1998). Using a similar methodology, Glenberg and Kaschak (2002) asked participants to discriminate between sensible sentences (e.g., "Open the drawer") and nonsense sentences (e.g., "Open the plate") using movements to a button close to the body or away from the body. They also observed an ACE in that responding was faster when the response required movement (e.g., toward the body) in a direction compatible with the direction implied by the sentence (e.g., opening a drawer generally requires movement toward the body). These data support the hypothesis that understanding a sentence requires consideration or simulation of the actions underlying the sentence. Glenberg and Kaschak also reported some evidence that people engage in bodily simulation even when understanding abstract ideas that do not directly correspond to actions.

The four experiments reported next use the ACE methodology to extend this work in several directions. First, we ask if language comprehension is sensitive to episodic affordances, for example, affordances that change with the location of the object relative to the actor. Some of the work cited above is consistent with this proposal, but none of it tested the proposal directly. Second, we determine if this episodic ACE requires sentences that describe or suggest action (as in Glenberg and Kaschak 2002), or whether it can be found with descriptive sentences as in Borghi and colleagues (2004). Third, we ask if the ACE depends on the objects being literally available for interaction, or whether the effect can also be found when the objects are no longer literally available, thereby implicating the use of perceptual symbols in the derivation of episodic affordances. Fourth, as in Borghi and colleagues we will determine if the ACE 
reflects action-based information (such as affordances) or spatial location. Fifth, we will show that the ACE is language-independent by replicating the main results of Experiment 1 with sentences written in German.

\section{Experiment 1}

\subsection{Participants}

A total of 51 participants were recruited from introductory psychology classes at the University of Wisconsin-Madison, and they were given partial course credit for their participation. Participants were treated in accordance with the ethical guidelines of the American Psychological Association.

\subsection{Design and materials}

All participants read a sentence centered on a computer screen, and they determined if the sentence was about a normal object or an odd one. There were several between subjects variables that were counterbalanced. For Response Direction, half the participants responded "Normal" by moving the right hand off a centered start key and then to the left, and they responded "Odd" by moving to a key on the right. The other half of the participants had the reverse assignment. There were two sets of six objects each, and each object had a particular part referred to in some of the sentences. Set A objects (and parts) were: apple (stem), pen (clip), shoe (heel), cup (handle), glasses (earpiece), bottle (neck). Set B objects were flower (leaf), watch (wristband), cell phone (antenna), knife (blade), nail (tip), and teddy bear (ear). For the independent variable Visible Set, half the participants had Set A objects arrayed in front of them, and half had Set B objects in front of them. The remaining objects were not visible. For the Left Objects variable, for half of the participants, the first three objects were arrayed on the left (e.g, apple, pen, and shoe in Set A) and the remaining three objects were on the right, and for half of the participants the arrangement was reversed. There were also several withinsubjects variables. For Sentence-Action, half of the sentences described a hand/arm action (hold, touch, press, grab, pick-up, and pull) that could be made in regard to an object using the object's named part (e.g, "Hold the apple by the stem"), and half of the sentences described an attribute of the object (e.g., "The apple is red"). For Sentence-Normalcy, twothirds of the sentences described a normal version of an object, and onethird of the sentences described an unusual or odd version of the object (e.g., "Hold the wristband of the apple" and "The apple has an antenna"). The odd objects were described using components from the nor- 
mal objects. Each object name appeared in 18 sentences, once with each of the six action verbs, once in six different descriptive sentences, in three sentences using an action verb and an odd object, and in three sentences describing an odd object. Because there were 12 objects there was a total of 216 sentences.

\subsection{Procedure}

Participants signed consent forms and then were directed to examine, pick up, and replace (on Velcro tabs) each of the six visible objects. Participants were then instructed on how to press the Start key with the index finger of the right hand to initiate presentation of a sentence, and how to move the same hand to either the Normal or Odd keys. Furthermore, they were instructed to read the sentence completely while holding the Start key because the sentence would disappear as soon as the key was released. Participants received 20 practice trials during which pressing the Start key presented the word "Normal" or "Odd" and the participant was to move to the appropriate response key. Next, participants practiced reading, judging, and responding to 10 sentences naming objects that were otherwise not mentioned in any sentence. Finally, the 216 experimental sentences were displayed in a quasi-random order that differed for each participant. For each sentence, we recorded both the time between pressing the start key and releasing the start key (the reading time, RT) and the response key pressed (Normal or Odd). We also recorded the movement time (time between releasing the start key and depressing a response key). Movement time was used only in the trimming procedure described next.

\subsection{Results and discussion}

The data from two subjects with very high error rates (e.g., about $90 \%$ errors; probably because of a miscoding in the data file) were eliminated. We then examined the difference in error rates between the Normal and Odd sentences because a large difference indicates that the participant was likely biased toward either the Normal or Odd response. We looked for a discontinuity in the distribution of differences and eliminated participants with differences above the discontinuity. In Experiment 1 we eliminated 10 participants with a difference in error rates of 0.10 or greater, leaving a total of 39 participants. (The discontinuity criterion varied across experiments from 0.06 to 0.15 .)

We performed analyses for the Normal sentences referring to the visible objects, that is, the sentences for which we predicted an ACE. In these analyses, we disregarded whether Object Set A or B was the visible set, 
and we collapsed the independent variables Response Direction and Left Object into one variable called Compatibility. For this variable, the direction of responding and the object location were either Compatible (e.g, when the sentence is about a normal object, the object is on the left, and the normal response key is on the left) or Incompatible (e.g., when the sentence is about a normal object, the object is on the left, and the normal response key is on the right). The ACE corresponds to a main effect of Compatibility. Thus, the variables in the analysis were Compatibility and Sentence-Action (whether the sentence describes an object or describes an action on the object). The important means are in Table 1. A type 1 error rate of 0.05 was used in all analyses. We report all significant results for these items. Following the advice of Raaijmakers and colleagues (1999) for designs such as those used in these experiments, we report only $F$-statistics based on subject variability.

The most important finding is the significant ACE (Compatibility effect) of $56 \mathrm{msec}, F(1,38)=5.83, M s e=20742$. That is, people were faster to respond correctly when the correct response (Normal, for these sentences) requires movement toward the same side as the location of the object. The size of the ACE was similar for the Action sentences $(63 \mathrm{msec})$ and the Descriptive sentences $(49 \mathrm{msec})$, and the interaction of Compatibility and Sentence-Action was not significant, $F<1$. There was also a main effect of Sentence-Action, $F(1,38)=38.91$, Mse $=24191$, at least in part because the Action sentences were on average several words longer than the Non-Action sentences.

There was also a significant effect in the analysis of error rates (see Table 1 for means), namely, the main effect of Compatibility, $F(1,38)=$ 7.26, $M s e=0.004$. Because there were more errors for the Incompatible condition than the Compatible condition, and the reading times were slower in the Incompatible condition, it is unlikely that the ACE reflects some sort of speed-accuracy tradeoff.

These results are exactly what were expected according to the IH and the proposition that language comprehension requires the derivation of

Table 1. Results from Experiment 1

\begin{tabular}{llllll}
\hline \multirow{2}{*}{ Compatibility } & \multicolumn{4}{c}{ Sentence-Action } \\
\cline { 2 - 3 } & \multicolumn{2}{c}{ Action } & & \multicolumn{2}{c}{ Descriptive } \\
\cline { 2 - 5 } & Error rate & $\mathrm{RT}$ & & Error rate & $\mathrm{RT}$ \\
\hline Incompatible & .07 & 1533 & & .06 & 1371 \\
Compatible & .03 & 1470 & .04 & 1322 \\
\hline
\end{tabular}


episodic affordances. That is, in understanding a sentence such as "Touch the apple by the stem," people index terms such as "the apple" to the actual apple in the environment. Then, the affordances of this particular apple, that is, how to interact with it, become relevant. In this case, location of the apple determines whether interaction requires movement to the left or right. When that direction matches the direction required to make the Normal response, responding is facilitated relative to when the directions mismatch. Importantly, the ACE is found for both Action sentences and Descriptive sentences such as, "The apple is red." The correct response for descriptive sentences could always be determined by consulting a putative semantic memory representation. Apparently, however, the participants indexed the words to the objects and derived affordances, leading to the ACE.

\section{Experiment 2}

The IH and the notion of episodic affordances provide one explanation for the results of Experiment 1. There is at least one important alternative, however. Suppose that the participants oriented towards objects named in the sentence, and the orientation affected action independent of any language comprehension processes. For example, Grubb and Reed (2002) report that rotating the trunk of the body can facilitate responding to objects in the same direction and interfere with responding in the opposite direction. Consequently, the results of Experiment 1 do not require an explanation in terms of affordances being used in language comprehension. Instead, the compatibility effect may simply reflect the compatibility between a physical or attentional orientation and responding.

Experiment 2 was designed to provide a test of this alternative hypothesis. For each participant, the first quarter of the experiment (54 trials) followed the same procedure as Experiment 1 with the exception that the sentences were only about the visible objects (in Set A or Set B). For the second quarter of the experiment, the objects were removed, and thus there were no physical objects to orient toward. We reasoned that if participants were indexing the words to perceptual symbols derived from recent experience, then the perceptual symbols should include location information that would produce an ACE. For the third quarter of the experiment, the objects in the alternative set (Set B or Set A) were physically present and the sentences were only about those objects. Finally, in the fourth quarter of the experiment, those objects were removed. In short, will we observe an ACE when objects are physically present (replicating Experiment 1)? And, will we observe and ACE when the objects are not 
present, contrary to an orientation explanation, but consistent with a perceptual symbol interpretation?

The data can also be used to test another type of attention hypothesis, namely, a shift in attention to guide hand movements. First, consider that Richardson and Spivey (2000) have demonstrated that in a recall task people tend to look at locations that had held objects even when those objects are no longer present. Thus, participants in Experiment 2 might also orient attention to the locations of the removed objects. Second, Rizzolatti and colleagues (1987; see also, Rizzolatti et al. 1997) have demonstrated that reorienting attention across the visual vertical meridian incurs a cost. Thus, suppose that attention is directed toward the location of the removed items in order to guide hand movements to make the response. When the reorientation crosses the vertical meridian, as would be more likely in making an incompatible response than a compatible response, there is a cost associated with the reorientation that shows up as an ACE. Note that this hypothesis predicts an ACE in the movement times when visual guidance is necessary. If the ACE is found in the reading times of the sentences referring to the removed objects, then it is unlikely to be due to a shift in attention for hand movement guidance. That is, even if participants orient to the locations of the missing items (as in Richardson and Spivey 2000), there is no need to reorient, and indeed the participant cannot know what location to reorient toward until after the sentence is read and comprehended and reading time determined.

\subsection{Participants}

The 48 participants were selected as in Experiment 1.

\subsection{Materials}

The sentences and objects were the same as in Experiment 1.

\subsection{Design and procedure}

The design was similar to that of Experiment 1 except for three differences. First, there was no variable of Visible Set; that is, the sentences always referred to items that were (or had been) visible. Second, participants began with either Set A or Set B objects and the Set order was counterbalanced. Third, we introduced another independent variable, Objects-Present: For the first quarter of the trials, the referent objects (Set A or Set B) were Present, for the second quarter the objects were Removed; for the third quarter the alternative objects were Present, and for the last quarter these objects were Removed. 
Before the first quarter, participants picked up and replaced (on a Velcro tab) each of the six objects, practiced moving from the Start key to the Normal and Odd response keys, and practiced responding to Normal and Odd sentences. At the end of the first quarter of trials, the computer signaled the participant to call the Experimenter who removed the objects. The participants were not informed that the objects would be removed before this time. After the second quarter, the computer again signaled the participant to call the experimenter who positioned the second set of objects. The participant manipulated these objects before beginning the third quarter of the trials. After the third quarter, the second set of objects was removed.

\subsection{Results and discussion}

The same data trimming procedures were used as in Experiment 1, except the criterion for excluding participants for a difference in Normal and Odd error rates was set at 0.15 or greater where there seemed to be a cluster of participants different from the rest. In all, the data from five participants were eliminated, resulting in a final set of 43 participants (from the initial set of 48). The data of major interest are presented in Table 2.

The $54 \mathrm{msec}$ ACE (main effect of Compatibility) was significant, $F(1,42)=18.33$, Mse $=13620$. The interaction between Compatibility and Objects-Present was not significant, $F(1,42)<1$, indicating that the size of the ACE was comparable when the objects were Present $(71 \mathrm{msec})$ and when the objects were Removed (38 msec). However, the $71 \mathrm{msec}$ ACE is significant, $F(1,42)=14.67$, Mse $=14405$, and the $38 \mathrm{msec}$ ACE is not, $F(1,42)=1.99$, Mse $=30699$. At first glance, this may appear to be problematic. However, Experiment 3 provides a replication of the ACE effect in the Removed condition that is quite large (91 msecs), $F(1,57)=16.03$, Mse $=28644$. Thus, the evidence for an ACE with

Table 2. Results from Experiment 2

\begin{tabular}{|c|c|c|c|c|c|}
\hline \multirow[t]{3}{*}{ Objects-Present } & \multirow[t]{3}{*}{ Compatibility } & \multicolumn{4}{|c|}{ Sentence-Action } \\
\hline & & \multicolumn{2}{|c|}{ Action } & \multicolumn{2}{|c|}{ Descriptive } \\
\hline & & Error rate & RT & Error rate & RT \\
\hline \multirow[t]{2}{*}{ Present } & Incompatible & .11 & 1590 & .09 & 1453 \\
\hline & Compatible & .10 & 1536 & .06 & 1367 \\
\hline \multirow[t]{2}{*}{ Removed } & Incompatible & .10 & 1442 & .08 & 1358 \\
\hline & Compatible & .08 & 1426 & .09 & 1299 \\
\hline
\end{tabular}


objects removed (lack of an interaction of Compatibility and ObjectsPresent in Experiment 2, a significant effect of Compatibility in the Removed condition of Experiment 3 ) is, in total, reasonably strong. ${ }^{1}$

There was a significant effect of Sentence-Action, $F(1,42)=22.45$, $M s e=64084$ because the Action sentences tended to be several words longer than the Descriptive sentences. There were no significant effects in an analysis of error rates.

The ACE for the objects Present condition provided a replication of Experiment 1. Again, we conclude that episodic affordances are considered during language comprehension both for sentences that describe action and for sentences that do not. Importantly, the data from the objects Removed condition allow us to rule out the alternative that the ACE is due to (physical or attentional) orientation to a particular object or side rather than language comprehension. That is, when the objects are removed, participants have no reason to physically orient to a particular side. Instead, people understand the sentences by indexing the words (e.g., "the apple") to a perceptual symbol (rather than the object itself). Affordances are derived from this perceptual symbol, and because location of an object relative to an actor helps to determine the interactions with those objects, location affects the derivation of affordances. These affordances are revealed by the ACE. As noted in the introduction to this experiment, because we used reading time as a dependent variable, and not movement time, the data are also contrary to the hypothesis that the ACE reflects only a reorientation of attention to guide hand movements.

\section{Experiment 3}

The data from the first two experiments demonstrate effects of episodic information on language comprehension. But, can these effects be interpreted as due to affordances, that is, possibilities for interacting with objects, or might another type of information, such as spatial location,

1. Why might the effect be significant in Experiment 3 and not in Experiment 2? It might indicate that the effect is unreliable. However, given that the effect was so large in Experiment 3 and approached significance in Experiment 2, it seems more likely that the difference reflects sampling error contributing to a Type II statistical error in Experiment 2. Supporting this speculation, Cumming (2008) documents how $p$-values are remarkably inconsistent across exact replications. For example, Cumming reports on 25 simulations of an independent sample, two-group experiment, each with an effect size of .5 , and each with a sample size of 32 for each group. Over the 25 simulations, $p$ ranged from less than .001 to .759 ! Furthermore, 13 of the 25 simulations had $p>.05$. 
underlie the effect? Experiment 3 was undertaken to answer this question. We reasoned that if the ACE was due to affordances derived from the objects, then the effect will be large when the action required to make the response (moving to the Normal key) is compatible or not with the affordances derived from the object at a particular location. In contrast, suppose that the ACE were due to match or mismatch of spatial location of the object and spatial location of the response key. In this case, an ACE would be found whether or not there is action directed at a location; the mere fact that the response key was in a compatible or incompatible spatial location should produce an ACE.

In Experiment 3, participants used two response modes. The Movement mode was similar to that used in Experiments 1 and 2. The Location mode used the same spatial arrangement of Normal and Odd keys as in the Movement mode, however, the participant did not move his or her hand to the keys. Instead, one index finger rested on each of the keys (the assignment of left or right to Normal or Odd was counterbalanced between participants as in Experiments 1 and 2). In Experiment 3, all responding was done using a symmetric keyboard. The Normal and Odd keys were the far left and far right keys on the top row of the keyboard (tilde and del). We made this change so that other laboratories would be able to replicate our experiments without needing to construct a specialized response apparatus.

The design of the experiment also allows us to test another attention hypothesis. Suppose, as noted before, participants attend to objects mentioned in the sentence or to the locations of the removed objects as in Richardson and Spivey (2000). This allocation of attention might create a type of Simon effect (e.g., Simon 1990) in that people are attending to one spatial location (that of the object) but must make a response in a different spatial location. Perhaps it is this Simon effect that produces the ACE. In this case, the ACE should be found in both the Movement mode and the Location mode. If the ACE is found only in the Movement mode, we can rule out this version of the attention hypothesis.

\subsection{Participants}

The 65 participants were selected as in Experiment 1.

\subsection{Design and procedure}

Much like Experiment 2, the experiment was divided into quarters. In the first quarter, participants were randomly assigned to object Set A or Set B. Also randomly assigned was initial Response Mode (Movement or Location). The second quarter maintained the Object Set and Response 
Mode assignments, but the objects were removed (as in Experiment 2). For the third and fourth quarters, both the Object Set assignment and the Response Mode assignment were changed to the other alternatives. The objects were Present in the third quarter and removed for the fourth quarter.

In the Movement response mode, participants used the right index or middle finger to first press the space bar and initiate presentation of the sentence. Next, they moved the right hand so that the index or middle finger would press either the Normal or Odd key. In the Location mode, the participant's hands rested on the keyboard so that the thumbs were on the space bar and the middle fingers rested on the Normal and Odd keys. The participant pressed the space bar to initiate presentation of the sentence and then used the middle finger to make the Normal or Odd response. Note that unlike the Movement condition, the participant did not have to move his or her hands to a new location to make the judgment.

\subsection{Results and discussion}

The data from one participant were eliminated for an error rate in excess of 15 percent. The data from six other participants were eliminated for having a difference between Normal and Odd sentence error rates of more than 6 percent (where there appeared to be a cluster of participants different from the majority). For the remaining 58 participants, all error trials were eliminated before analyzing the reaction times. The data of major interest are in Table 3.

Table 3. Results from Experiment 3

\begin{tabular}{|c|c|c|c|c|c|c|}
\hline \multirow[t]{3}{*}{ Response Mode } & \multirow[t]{3}{*}{ Objects- Present } & \multirow[t]{3}{*}{ Compatibility } & \multicolumn{4}{|c|}{ Sentence-Action } \\
\hline & & & \multicolumn{2}{|c|}{ Action } & \multicolumn{2}{|c|}{ Description } \\
\hline & & & $\begin{array}{l}\text { Error } \\
\text { rate }\end{array}$ & RT & $\begin{array}{l}\text { Error } \\
\text { rate }\end{array}$ & RT \\
\hline \multirow[t]{4}{*}{ Movement } & \multirow[t]{2}{*}{ Present } & Incompatible & .08 & 1875 & .06 & 1677 \\
\hline & & Compatible & .06 & 1744 & .04 & 1629 \\
\hline & \multirow[t]{2}{*}{ Removed } & Incompatible & .03 & 1595 & .08 & 1600 \\
\hline & & Compatible & .02 & 1552 & .04 & 1461 \\
\hline \multirow[t]{4}{*}{ Location } & \multirow[t]{2}{*}{ Present } & Incompatible & .07 & 1631 & .07 & 1503 \\
\hline & & Compatible & .06 & 1664 & .06 & 1476 \\
\hline & \multirow[t]{2}{*}{ Removed } & Incompatible & .05 & 1392 & .07 & 1400 \\
\hline & & Compatible & .03 & 1382 & .05 & 1339 \\
\hline
\end{tabular}


In replication of Experiments 1 and 2, a 53 msec ACE (main effect of Compatibility) was significant, $F(1,57)=15.36, M s e=42510$. Also as in Experiment 2, there was no interaction between Compatibility and Objects-Present, $F(1,57)=0.73, M s e=31043$, indicating a substantial ACE effect for both objects Present $(43 \mathrm{msec})$ and objects Removed (63 msec).

The most important effect for this experiment is the interaction between Response Mode and Compatibility. That is, does the size of the ACE change with the response mode? The answer is "yes", as indicated by the within-a-hair's-breadth-significant interaction, $F(1,57)=3.63$, $M s e=87282, p=0.06$. In the Movement Response Mode, the $90 \mathrm{msec}$ ACE was significant, $t(57)=3.49, S E=26$, whereas in the Location Response Mode, the $16 \mathrm{msec}$ ACE was not significant, $t(57)=.76, S E=21$. Apparently, the ACE reflects derivation of affordances because it is robust when there is real action, but the effect is much reduced when the incompatibility is between spatial locations. Furthermore, given that the ACE is not significant in the Location Response Mode, we can rule out the version of the attention hypothesis described in the introduction to this experiment.

There were several other significant effects, although they do not appear to compromise the main conclusions. There was a significant effect of Sentence-Action, $F(1,57)=23.47$, Mse $=86818$, because the Action sentences tended to be several words longer than the Descriptive sentences. There was also a main effect of Objects-Present, $F(1,57)=88.45$, $M s e=89270$. Responding was slower when the objects were Present compared to Removed, probably because of practice effects (the objects were always Present before they were Removed). There was also a significant interaction between Sentence-Action and Objects-Present, $F(1,57)=$ 19.05, Mse $=48737$. In the Objects-Present condition, the Descriptive sentences were read $157 \mathrm{msec}$ faster than the Action sentences, whereas in the Objects-Absent condition this difference was only $30 \mathrm{msec}$. This interaction is also likely to be due to practice effects. That is, after practice with the names of the objects, parts, and verbs, the difference in length between the Action and Descriptive sentences did not count for as much.

In the analysis of error rates, there was a significant effect of Compatibility, $F(1,57)=11.66$, Mse $=0.006$, and a significant effect of ObjectsPresent, $F(1,57)=5.03$, Mse $=0.008$. There was also a significant interaction between Objects-Present and Sentence-Action, $F(1,57)=17.69$, Mse $=0.006$. Importantly, there was no hint of an interaction between Movement Condition and Compatibility that might have compromised the interpretation of the reaction time data, $F(1,57)=0.61, M s e=0.005$. 


\section{Experiment 4}

Experiment 4 was a close replication of Experiment 1 that differed in two ways. First, the sentences were translated into German, and the participants were native German speakers. Second, the response apparatus was a keyboard, much like in Experiment 3. Experiment 4 was conducted for two reasons. First, by providing a cross-language replication, it indicates that the derivation and use of episodic affordances is not peculiar to English or proclivities of American students. Second, when compared to an unreported experiment, it reinforces the conclusion that the ACE arises from action systems, not just spatial relations. The unreported experiment was also a replication of Experiment 1 using German sentences. However, the response apparatus was a joystick that could be moved from side to side by turning the wrist. In that experiment, there was nary a hint of an ACE. ${ }^{2}$ On reflection, we realized that the wrist movements were quite different (in extent, direction - they were rotational - and effector systems) from those that would be used to literally interact with the objects on either side of the participant. Consequently, we ran the experiment again using a keyboard which more closely mimics the actions required to interact with the objects (i.e., the hand is lifted and moved to the side).

\subsection{Participants}

The 64 German participants were recruited from introductory psychology classes at Dresden University of Technology, and they were given partial course credit for their participation.

\subsection{Materials}

The objects and the contents of the sentences were the same as in Experiment 1, except that the sentences were translated into German. For instance, the sentence "Hold the apple by the stem" was translated as "Hebe den Apfel am Stiel an".

\subsection{Design and procedure}

The design and procedure were equivalent to Experiment 1, except that a keyboard was used as the response apparatus.

2. Twenty-seven German psychology students participated in this experiment. Their joystick movement times for normal sentences referring to visible objects yielded an insignificant ACE of $-28 \mathrm{msec}$, ACE $F(1,26)=1.04, M s e=22444$. Error rates did not yield any significant effects either, averaging only $4 \%$. 
Table 4. Results from Experiment 4

\begin{tabular}{llllll}
\hline \multirow{2}{*}{ Compatibility } & \multicolumn{4}{c}{ Sentence-Action } \\
\cline { 2 - 3 } & \multicolumn{2}{c}{ Action } & & \multicolumn{2}{c}{ Descriptive } \\
\cline { 2 - 3 } & Error Rate & RT & & Error Rate & RT \\
\hline Incompatible & .08 & 1406 & .04 & 1162 \\
Compatible & .07 & 1284 & .04 & 1079 \\
\hline
\end{tabular}

\subsection{Results and discussion}

The same data trimming procedures were used as in Experiment 1, leading to the exclusion of two participants with unusually high error rates. The data of major interest are presented in Table 4. Replicating Experiment 1 , we found a significant Compatibility effect of $102 \mathrm{msec}$, $F(1,61)=7.73, M s e=646783$. That is, people were faster to respond correctly when the correct response (Normal, for these sentences) required movement toward the same side as the location of the object. The size of the Compatibility effect was similar for the Action sentences (122 msec) and the Descriptive sentences $(83 \mathrm{msec})$, and the interaction of Compatibility and Sentence-Action had an $F<1$. The Compatibility effect was also significant for Action and Descriptive sentences separately, with both $t \mathrm{~s}(61)>2.09$. There were no significant effects in the analysis of error rates (see Table 4 for means).

These results replicate those of Experiment 1 closely, thus demonstrating that the ACE observed in the previous experiments is not specific to the English language. This finding is consistent with our hypothesis that language comprehension requires the derivation of episodic affordances in any language. In addition, the contrast between these results and the failure to find an ACE in an identical experiment using a joystick is consistent with the claim (from Experiment 3) that the ACE reflects action coding of sentences, not simply spatial location.

\section{General discussion}

The data from the four experiments present a consistent picture. First, an ACE was found in all experiments, and it was found for both Action sentences and Descriptive sentences, respectively, $63 \mathrm{msec}$ and $49 \mathrm{msec}$ (Experiment 1), $35 \mathrm{msec}$ and $72 \mathrm{msec}$ (Experiment 2), $87 \mathrm{msec}$ and $94 \mathrm{msec}$ (Experiment 3, Movement condition), and $122 \mathrm{msec}$ and $83 \mathrm{msec}$ (Experiment 4). Finding an ACE for the descriptive sentences is consistent with work of Tucker and Ellis (1998) and Borghi and colleagues (2004), both 
of whom found evidence for automatic encoding of action components when people observed pictures of the objects (Tucker and Ellis) or printed words (Borghi et al.) without any requirement to overtly interact with the objects. Similarly, Pulvermüller (2008) reports the automatic activation of motor cortex upon perceiving action verbs when there is no requirement to act. Thus, we can be relatively confident that the processing of language for meaning activates information about action.

The results are also consistent in showing that the activation of action information does not depend on the object or a picture being physically available. The ACE for the Present and Removed conditions, respectively, were $70 \mathrm{msec}$ and $38 \mathrm{msec}$ (Experiment 2; the latter is not statistically significant, but see footnote 1) and $90 \mathrm{msec}$ and $91 \mathrm{msec}$ (Experiment 3, Movement condition). Thus, action encoding is characteristic of perceptual symbols (e.g., Barsalou 1999) as well as actual objects.

In contrast to this consistency, changing the response mode greatly reduced or eliminated the ACE. In the Location condition of Experiment 3, the ACE was a non-significant $16 \mathrm{msec}$, and in the experiment using a joystick (see footnote 2), it was a non-significant $-28 \mathrm{msec}$. This contrast helps to rule out several alternative explanations. First, the ACE is unlikely to be a spatial compatibility effect or Simon effect (Simon 1990). That is, if the ACE arose from a mismatch between the (irrelevant) location of the object referred to in the sentence and the location of the response, it should be found whether the response requires movement or not.

The contrast in response modes also helps to rule out several (if not all) variants of attentional hypotheses. Thus, the ACE is unlikely to be due to physical orientation to the objects because a) it is found when the objects are removed and b) it is not found when the objects are present but there is no requirement to move to make the response ( $-3 \mathrm{msec}$ ACE in Location condition, objects present). Also, it is unlikely that the ACE reflects a simple covert attention to the location of the items (whether present or removed) as demonstrated by Richardson and Spivey (2000). If the effect was due to covert attention to the location, the effect should be found for both the Movement and Location response modes. Note that we are not suggesting that people do not covertly attend to locations in which objects were previously displayed. We are suggesting, however, that an attentional mechanism without regard to action is not sufficient to account for the ACE. One other attentional account that is weakened by the pattern of data is the suggestion that ACE reflects covert attention to spatial location conjoined with a movement of attention needed to guide hand movements. This account has difficulty explaining the finding that the ACE was measured by reading time before the necessity for any movement. 
Might some form of spatial working memory suffice to explain the effects in the objects-removed conditions of Experiments 2 and 3? This account also seems unlikely if one conceives of spatial working memory as holding abstract representation. That is, such a representation has no obvious way of accounting for the absence of the effect in the Location Response Mode of Experiment 3. Whatever is producing the ACE, it must be specific to action, not just spatial location.

One might still argue that attention shifted in anticipation of the hand movement, thus, hypothetically, the ACE arises because of a) covert shift in attention to the location of objects that are not present and b) a shift in attention in anticipation of guidance of hand movements. While logically possible, this alternative appears more complex (two covert processes neither of which is required by the reading task) than the explanation favored here: The ACE reflects access of action-related components of meaning, and in the incompatible condition those components interfere with the need to move in the opposite direction.

To spell out this account more fully, we propose the following (as based on the Indexical Hypothesis). First, participants index words or phrases (e.g., "the apple") to actual objects or their perceptual symbols. Second, affordances are derived from these objects. It is at this stage that the data from the four experiments help to flesh out the theoretical claims. The data support the claim that affordances are a component of the information derived whether or not one intends to literally act on the objects. Furthermore, these affordances are not only those that might be stored in long-term memory. Instead, the affordances take into account current or recent experience with the objects. Third, as described in Kaschak and Glenberg (2000) and de Vega and colleagues (2004), but not tested here, the affordances are combined as specified by syntax. Finally, the processes of deriving and combining affordances require premotor cortex (Rizzolatti and Arbib 1998) and motor cortex (Hauk et al. 2004). The use of these systems to simulate the meaning of the sentences interferes with the literal movement required to make the response, thus resulting in the ACE. Note that by this account, perceptual symbols correspond to an active simulation that is based on both perceptual information and actions consistent with that information.

Several aspects of the claims made in this article may seem unusual. Here we discuss how these claims are not just supported by the empirical data but also make theoretical and practical sense. Consider first how skill in indexing is of benefit to language users. According to many older (Collins and Loftus 1975; Kintsch 1988) and more recent (Burgress and Lund 1997; Landauer and Dumais 1997; Vigliocco et al. 2004) theories of meaning and language, the meaning of a word (e.g, "chair") is 
captured by features stored in memory. Consequently, it is difficult to extend the use of these words to new, unanticipated situations, except perhaps through a process of inference and analogy. In contrast, consider the flexibility of indexing and using characteristics of the indexed object (or perceptual symbol) as the meaning. Indexing a word such as "chair" at different times to a kitchen chair, a throne, a dollhouse chair, or a log near a campfire, allows very different properties to become relevant to comprehension. Thus, a sentence about standing on a chair to change a light bulb in a ceiling fixture is sensible when the chair is a kitchen chair, but not when it is a dollhouse chair; or one can count the rings to determine the age of the chair near the campfire, but not the chair in the palace. Thus, indexing allows a limited set of words to be used flexibly in different situations to address the needs of communication within that situation.

The flexibility in indexing runs the risk of confusion, that is, the speaker may index a word to one thing (e.g., a kitchen chair) and the listener may index the word to something quite different (e.g., a log). This potential for confusion is greatly reduced by the highly practiced (Clark 1996) and virtually automatic (Clark 1996; Kelly et al. 1999; Pickering and Garrod 2004) procedures for establishing common ground. Thus, indexing allows effective "off-loading" of cognition to the environment (Wilson 2002). That is, rather than storing all possible meanings and features of words in memory, the relevant features are derived, when needed, from the situation at hand.

Presuming that indexing does occur, why should anything like affordances be derived from the objects or perceptual symbols? There are at least two answers to this question. The first relates to the symbol grounding problem. Searle (1980), Harnad (1990) and others have argued that symbols (e.g., words) can only be meaningful when they are grounded in something outside of the symbol system. Action provides a compelling domain in which to ground meaning (Glenberg 1997; Tucker and Ellis 1998). Here we develop a different line of reasoning, namely that derivation and combination of affordances can serve the metacognitive purpose of detecting when one has improperly indexed a term, and thus when one is not adequately understanding what the speaker or writer is trying to convey. The key idea is that affordances are not arbitrary, nor can they be arbitrarily combined. Consider understanding an imperative such as "Stand on the chair to change the light bulb in the ceiling fixture." If a listener were to index "the chair" to a dollhouse chair (perhaps because of recently playing with a child and a dollhouse), the listener would be puzzled because the dollhouse chair does not afford standing-on to raise the body. Note that this process does not require a sophisticated metacog- 
nitive analysis of the state of comprehension. Instead, failure to understand is immediately signaled by the inability to simulate (or carry out) the actions. When simulation runs afoul of affordances, one option is try to re-establish common ground by re-indexing.

How do these claims relate to the notion of episodic affordances? In some ways, the term "episodic" is redundant. That is, affordances refer to the relation between a particular type of body and a particular situation, and because bodies and situations are in constant flux, the affordances are too. We introduce the term to focus attention on the fact that although some affordances could be stored in memory, many need to be derived from the current situation. Although the experimental work focused on affordances related to spatial location, there may well be other factors that contribute to episodic affordances, for example, human goals. The relevant affordances of a (kitchen) chair depend on goals: does one need to sit, change a light bulb, hide in a game of hide-and-seek, or defend oneself against a snarling dog? Not all affordances are derived at any one time because most of those affordances would be irrelevant to the current situation and goals (see Kaschak and Glenberg 2000, for data supporting this contention). Thus, the properties of the situation that determine meaning change with physical properties of the situation, biological properties of the body, and psychological properties such as goals. Given that human goals are likely to be quite diverse, a communication system that can deal flexibly with this diversity is advantageous. Episodic affordances are one signature of this flexibility.

\section{References}

Barsalou, L. W. 1999. Perceptual symbol systems. Behavioral and Brain Sciences 22. 577660.

Borghi, A. M., A. M. Glenberg \& M. P. Kaschak. 2004. Putting words in perspective. Memory \& Cognition 32. 863-873.

Buccino, G., F. Binkofski, V. Gallese, G. Melli, L. Riggio \& G. Rizzolatti. 2005. Listening to action-related sentences modulates the activity of the motor system: A combined TMS and behavioral study. Cognitive Brain Research 24. 355-363.

Buccino, G., F. Benuzzi, G. Lagravinese, F. Lui, I. Pateri, C. A. Porro \& G. Rizzolatti. 2004. Neural circuits involved in the recognition of actions performed by nonconspecifics: An fMRI study. Journal of Cognitive Neuroscience 16. 114-126.

Burgess, C. \& K. Lund. 1997. Modelling parsing constraints with high-dimensional context space. Language \& Cognitive Processes 12. 177-210.

Chambers, C. G., G. N. Carlson, K. M. Eberhard, H. Filip \& M. K. Tanenhaus. 2002. Circumscribing referential domains during real-time language comprehension. Journal of Memory and Language 47. 30-49.

Chambers, C. G., J. S. Magnuson \& M. K. Tanenhaus 2004. Actions and affordances in syntactic ambiguity resolution. Journal of Memory and Language 30. 687-696.

Clark, H. H. 1996. Using language. New York: Cambridge University Press. 


\section{A. M. Glenberg et al.}

Collins, A. M. \& E. F. Loftus. 1975. Spreading activation theory of semantic memory. Psychological Review 82. 407-428.

Coventry, K. \& S. C. Garrod 2003. Saying, seeing, and acting: The psychological semantics of spatial prepositions. New York: The Psychology Press.

Cumming, G. 2008. Replication and $p$ intervals: $p$ values predict the future only vaguely, but confidence intervals do much better. Perspectives on Psychological Science 3. 286-300.

De Vega, M., A. M. Glenberg, M. P. Kaschak, M. Rinck \& D. A. Robertson. 2004. On doing two things at once: Temporal constraints on Actions in language comprehension. Memory and Cognition 32. 1033-1043.

Gibson, J. J. 1979. The ecological approach to visual perception. New York: Houghton Mifflin.

Glenberg, A. M. 1997. What memory is for. Behavioral and Brain Sciences 20. 1-19.

Glenberg, A. M., G. Buccino, L. Cattaneo, D. Palumbo, L. Riggio \& M. Sato. 2008. Processing abstract language modulates motor system activity. Quarterly Journal of Experimental Psychology 61. 905-919.

Glenberg, A. M. \& M. P. Kaschak. 2002. Grounding language in action. Psychonomic Bulletin \& Review 9. 558-565.

Glenberg, A. M. \& D. A. Robertson. 1999. Indexical understanding of instructions. Discourse Processes 28. 1-26.

Glenberg, A. M. \& D. A. Robertson. 2000. Symbol grounding and meaning: A comparison of high-dimensional and embodied theories of meaning. Journal of Memory and Language 43. 379-401.

Grubb, J. D. \& C. L. Reed. 2002. Trunk orientation induces neglect-like lateral biases in covert attention. Psychological Science 13. 553-556.

Harnad, S. 1990. The symbol grounding problem. Physica D 42. 335-346.

Hauk, O., I. Johnsrude \& F. Pulvermüller. 2004. Somatotopic representation of action words in human motor and premotor cortex. Neuron 41. 301-307.

Kaschak, M. P. \& A. M. Glenberg. 2000. Constructing meaning: The role of affordances and grammatical constructions in sentence comprehension. Journal of Memory and Language 43. 508-529.

Kamide, Y., G. T. M. Altmann \& S. L. Haywood. 2003. The time-course of prediction in incremental sentence processing: Evidence from anticipatory eye movements. Journal of Memory and Language 49. 133-156.

Kelly, S. D., D. J. Barr, R. B. Church \& K. Lynch. 1999. Offering a hand to pragmatic understanding: The role of speech and gesture in comprehension and memory. Journal of Memory and Language 40. 577-592.

Kintsch, W. 1988. The role of knowledge in discourse comprehension: A constructionintegration model. Psychological Review 95. 163-182.

Lakoff, G. 1987. Women, fire, and dangerous things: What categories reveal about the mind. Chicago: University of Chicago Press.

Landauer, T. K. \& S. T. Dumais. 1997. A solution to Plato's problem: The Latent Semantic Analysis theory of acquisition, induction and representation of knowledge. Psychological Review 104. 211-240.

Pickering, M. J. \& S. Garrod. 2004. Toward a mechanistic psychology of dialogue. Behavioral and Brain Sciences 22. 169-225.

Pulvermüller, F. 2008. Grounding language in the brain. In M. de Vega, A. M. Glenberg, \& A. C. Graesser (eds.), Symbols, embodiment, and meaning, 85-116. Oxford: Oxford University Press.

Raaijmakers, J. G., F. Gremmen \& J. M. C. Schrijnemakers. 1999. How to deal with 'The language-as-fixed-effect fallacy': Common misconceptions and alternative solutions. Journal of Memory and Language 41. 416-426.

\section{Author's Copy}


Richardson, D. C. \& M. J. Spivey. 2000. Representation, space and Hollywood Squares: Looking at things that aren't there anymore. Cognition 76. 269-295.

Rizzolatti, G. \& M. A. Arbib. 1998. Language within our grasp. Trends in Neuroscience 21. 188-194.

Rizzolatti, G., I. Dascola, L. Riggio \& C. Umiltà. 1987. Reorienting attention across the horizontal and vertical meridians: Evidence in favor of a premotor theory of attention. Neuropsychologia 25. 31-40.

Rizzolatti, G., L. Fadiga, L. Fogassi \& V. Gallese. 1997. The space around us. Science 277. 190-191.

Searle, J. R. 1980. Minds, brains and programs. Behavioral and Brain Sciences 3. 417-424.

Simon, J. R. 1990. The effects of an irrelevant directional cue on human information processing. In R. W. Proctor and T. G. Reeve (eds.), Stimulus-response compatibility: An integrated perspective, 31-86. Amsterdam: North-Holland.

Tucker, M. \& R. Ellis. 1998. On the relations between seen objects and components of potential actions. Journal of Experimental Psychology: Human Perception and Performance 24. $930-846$.

Vigliocco, G., M. F. Garrett, W. Lewis \& D. P. Vinson. 2004. Representing the meanings of object and action words: The featural and unitary semantic space hypothesis. Cognitive Psychology 48. 422-488.

Wilson, M. 2002. Six views of embodied cognition. Psychonomic Bulletin \& Review 9. 625636. 